\title{
Sleep-disordered breathing in deep vein thrombosis and acute pulmonary embolism
}

\author{
Michael Arzt*, Ruth Luigart*, Carola Schum*, Lars Lüthje”, Annika Stein\#, \\ Iris Koper ${ }^{*}$, Cornelia Hecker ${ }^{+}$, Rio Dumitrascu ${ }^{+}$and Richard Schulz ${ }^{+}$, \\ for the "Circulation and Sleep" working group of the German Society of Sleep \\ Research and Sleep Medicine (DGSM)
}

ABSTRACT: Sleep-disordered breathing (SDB) may trigger thromboembolic events by enhancing intravascular clot formation. The primary objective of the present nested case-control study was to investigate whether the prevalence of SDB is increased in patients with deep vein thrombosis (DVT) and/or acute pulmonary embolism (PE).

82 consecutive patients with DVT and/or PE (cases) were prospectively enrolled irrespective of SDB-related symptoms and formed matched pairs with patients without DVT and/or PE (controls) according to sex and pre-defined categories of age and body mass index.

The prevalence of SDB (respiratory disturbance index assessed by polygraphy $\geqslant 15$ events $\cdot h^{-1}$ ) was significantly greater in the cases with DVT and/or PE than in controls (40 versus $26 \%, p=0.046$ ) and was predominantly obstructive in nature. Multiple regression analysis revealed that SDB was significantly associated with DVT and/or PE (OR 2.28, 95\% CI 1.08-4.85; $p=0.032$ ) independent of established risk factors for thrombosis. In the sex-specific analyses this association was significant in females (OR 4.14, 95\% Cl 1.05-16.36; $\mathrm{p}=0.042$ ), but not in males (OR $1.55,95 \% \mathrm{Cl} 0.57-4.21$; $p=0.391)$.

SDB occurs more frequently in females with DVT and/or PE than in controls matched for anthropometric variables, and is independently associated with the occurrence of these thromboembolic events.

KEYWORDS: Pulmonary embolism, sleep apnoea, thrombosis

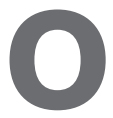
bstructive sleep apnoea (OSA) is a highly prevalent sleep-related breathing disorder. Apart from causing poor sleep quality and daytime sleepiness, it is recognised as an independent risk factor for cardio- and cerebrovascular disease, such as arterial hypertension, coronary artery disease, congestive heart failure and stroke. OSA has also been suggested to increase the risk for thromboembolic disease such as deep vein thrombosis (DVT) and acute pulmonary embolism (PE). This assumption is primarily based on the fact that OSA is associated with a variety of haemostatic alterations which may set the stage for a pro-thrombotic milieu [1].

In line with this hypothesis, the prevalence of sleep-disordered breathing (SDB) has already been reported to be increased in patients with DVT and/or PE [2-4]. However, two of these studies [2, 3] had some important methodological drawbacks, such as retrospective design, lack of a control group without DVT and/or PE, or diagnosis of SDB only by patient history.
The present study by the "Circulation and Sleep" working group of the German Society of Sleep Research and Sleep Medicine (DGSM) was designed to overcome such limitations. The aims were to investigate the prevalence and type of SDB in patients with DVT and/or PE versus controls, and to determine if it is significantly associated with prevalent DVT and/or PE independent of established risk factors for thromboembolic events.

\section{PATIENTS AND METHODS}

The patients participating in the present study were prospectively enrolled at four medical centres in Germany. All index patients had been consecutively admitted to the hospitals with the clinical suspicion of DVT and/or acute PE, and the diagnosis had been verified by duplex sonography/phlebography and/or chest computed tomography (CT) scan/ ventilation-perfusion scintigraphy, respectively, according to current guidelines on the management of DVT and PE [5]. Exclusion criteria were the following: age $<18$ or $>80$ yrs, known SDB
Recruitment of cases and controls

\section{AFFILIATIONS}

*Dept of Internal Medicine II, Pneumology, University Hospital Regensburg, Regensburg, "Dept of Internal Medicine, Cardiology, University Hospital Göttingen, Göttingen,

"Clinic Oldenburg/Ostholstein, Oldenburg, and

+Lung Center, University of Giessen, Giessen, Germany.

CORRESPONDENCE

M. Arzt

Center for Sleep Medicine Dept of Internal Medicine II University Hospital Regensburg Franz-Josef-Strauss-Allee 11 93053 Regensburg Germany E-mail: michael.arzt@klinik.uniregensburg.de

Received:

Oct 112011

Accepted after revision: Dec 162011 First published online: Feb 232012 
and the presence of major PE with arterial hypoxaemia (i.e. dependence on nasal oxygen) and/or haemodynamic instability (i.e. systolic blood pressure $<100 \mathrm{mmHg}$ ). Finally, patients with pre-existing congestive heart failure with a left ventricular ejection fraction $<50 \%$ as determined by echocardiography were not allowed to enter the study. The control subjects without current or prior DVT and/or PE were recruited from the pool of patients currently hospitalised in the same institution as the cases (nested case-control design), and were matched with them 1:1 with regard to sex and pre-defined categories of age and body mass index (BMI) (table 1).

Patient recruitment was independent of the presence of SDBrelated symptoms (i.e. snoring, witnessed apnoeas and excessive daytime sleepiness) and known risk factors for thrombosis (i.e. immobilisation, surgery or trauma within the preceding 3 months, cancer, smoking, oral contraceptives, and a positive family history of DVT and/or PE).

The study was started in June 2009 and completed in November 2010. Its protocol was approved by the local ethics committees and all individuals gave their informed written consent.

\section{Assessment of cases and controls}

The anthropometric parameters of the patients were determined, i.e. age, sex and BMI. After inclusion into the study, all participants completed the Epworth Sleepiness Scale to assess their subjective level of daytime sleepiness and were evaluated for the presence of the aforementioned risk factors for thrombosis.

\section{Polygraphy}

Polygraphy was performed within 4 weeks of the diagnosis of DVT and/or PE. The Stardust II system (Respironics Inc., Herrsching, Germany) was used for polygraphy. This device records arterial oxygen saturation $\left(\mathrm{Sp}_{\mathrm{p}} \mathrm{O}_{2}\right)$ and heart rate by fingertip pulse oximetry. Airflow was monitored by a pressure cannula attached to the patient's nostrils, breathing efforts were measured by an inductive plethysmographic belt tightly wrapped around the chest and, finally, body position was registered. Apnoeas and hypopnoeas were visually analysed according to the current American Academy of Sleep Medicine (AASM) guidelines by experienced investigators blinded to the clinical data [6]. Hypopnoea definition A was used [6]. A respiratory disturbance index (RDI) of $\geqslant 5, \geqslant 15$ and $\geqslant 30$ events $\cdot h^{-1}$ recording time was considered as diagnostic of an at least mild, moderate and severe degree of SDB, respectively.

\begin{tabular}{|c|c|c|c|}
\hline TABLE 1 & $\begin{array}{l}\text { Pre-define } \\
\text { controls } 1\end{array}$ & gories fo & ig cases and \\
\hline Category & Sex & Age yrs & $\mathrm{BMI} \mathbf{k g} \cdot \mathrm{m}^{-2}$ \\
\hline 1 & Female & $18-30$ & 15.0-20.0 \\
\hline 2 & Male & $31-40$ & $20.1-25.0$ \\
\hline 3 & & $41-50$ & $25.1-29.0$ \\
\hline 4 & & $51-60$ & $29.1-35.0$ \\
\hline 5 & & $61-70$ & $35.1-50.0$ \\
\hline 6 & & $71-80$ & \\
\hline
\end{tabular}

BMI: body mass index.

\section{Statistical analysis}

Baseline characteristics of groups were compared by the MannWhitney U-test or unpaired t-test for quantitative variables, or Chi-squared statistics for binary variables. To evaluate whether SDB (RDI $\geqslant 15$ events $\cdot h^{-1}$ ) is associated with prevalent DVT and/or PE independently of established predisposing factors, we performed a multivariate binary logistic regression analysis. SDB (RDI $\geqslant 15$ events $\cdot h^{-1}$ ), surgery or trauma, immobilisation, cancer, family history of DVT and/or PE were included in the model as independent variables. In the sex-specific subanalyses, models with additional adjustment for pregnancy and coronary artery disease were also performed.

Continuous variables are shown as median (interquartile range), or mean $\pm \mathrm{SD}$, depending on data distribution. A p-value $<0.05$ was considered significant. Statistical analyses were performed with PASW Statistics software version 18.0 (IBM Corp., Armonck, NY, USA).

\section{RESULTS}

\section{Case and control patients}

A total of 100 consecutive cases with DVT and/or PE, and 109 control participants without a thromboembolic event were evaluated for eligibility for the study. 18 cases had to be excluded from analyses because they refused to give informed consent $(n=5)$, or because of missing polygraphic data $(n=3)$, history of SDB $(n=2)$, congestive heart failure $(n=5)$ and no matching control subject $(n=3)$. 27 control participants had to be excluded due to history of DVT, PE $(n=5)$ or SDB $(n=1)$, missing polygraphic data $(n=1)$, congestive heart failure $(n=6)$ and no matching case subject $(n=14)$. Thus, 82 cases with DVT and/or $\mathrm{PE}$ and 82 age-, BMI- and sex-matched control patients were enrolled.

By design cases with DVT and/or PE had a very similar age and BMI compared with controls. On average, the study population was middle-aged and mildly overweight. In addition, the proportion of females was identical in both groups (table 2). The frequencies of thromboembolic events in the group of cases were $21 \%$ DVTs alone, 37\% PEs alone and 43\% DVTs with an acute PE (table 2). Cases with a DVT and/or PE had significantly more often a history of known risk factors for thromboembolic events, such as surgery or trauma $(p=0.001)$, immobilisation $(p<0.001)$, cancer $(p<0.001)$, previous DVT and/or PE $(p<0.001$ and $p=0.013$, respectively), and family history of DVT and/or PE $(\mathrm{p}=0.007)$ (table 2$)$. The proportion of active smokers and females taking oral contraceptives was similar in both groups $(p=0.550$ and $p=1.000$, respectively).

With respect to comorbidities, coronary artery disease was more frequent in the control group $(p=0.011)$, while the prevalence of arterial hypertension and chronic obstructive pulmonary disease was similar in both groups $(\mathrm{p}=0.257$ and 0.118 , respectively). The majority of patients in both groups were in sinus rhythm and the prevalence of atrial fibrillation was not significantly different between the case and the control groups $(\mathrm{p}=0.306)$ (table 3$)$.

In the sex-specific analyses of risk factors for DVT and/or PE and comorbidities, we found the following differences between males and females. 1) The prevalence of coronary artery disease was generally higher in males $(33 \%)$ than in females $(8 \%)$. In males, the prevalence of coronary artery disease was 


\begin{tabular}{|c|c|c|c|}
\hline \multirow[t]{2}{*}{ TABLE 2} & \multicolumn{3}{|c|}{$\begin{array}{l}\text { Characteristics of deep vein thrombosis (DVT)/ } \\
\text { pulmonary embolism (PE) cases and controls }\end{array}$} \\
\hline & DVT/PE cases & Controls & $\mathrm{p}$-value \\
\hline Subjects $n$ & 82 & 82 & \\
\hline \multicolumn{4}{|l|}{ Demographics } \\
\hline Age yrs & $57 \pm 17$ & $56 \pm 17$ & 0.864 \\
\hline $\mathrm{BMl} \mathrm{kg} \cdot \mathrm{m}^{-2}$ & $27.6 \pm 4.5$ & $27.5 \pm 4.4$ & 0.817 \\
\hline Female \% & 49 & 49 & 1.0 \\
\hline \multicolumn{4}{|l|}{ Thromboembolic events } \\
\hline DVT & $17(21)$ & & \\
\hline PE & $30(37)$ & & \\
\hline DVT and PE & $35(43)$ & & \\
\hline \multicolumn{4}{|l|}{$\begin{array}{l}\text { Risk factors for thrombo- } \\
\text { embolic events }\end{array}$} \\
\hline Surgery or trauma & $23(28)$ & 7 (9) & 0.001 \\
\hline Immobilisation & $23(28)$ & $3(4)$ & $<0.001$ \\
\hline Cancer & $21(26)$ & $5(6)$ & $<0.001$ \\
\hline History of DVT & $20(24)$ & $0(0)$ & $<0.001$ \\
\hline History of PE & $6(7)$ & $0(0)$ & 0.013 \\
\hline Family history of DVT/PE & $21(26)$ & $8(10)$ & 0.007 \\
\hline Smoking & $17(21)$ & $14(17)$ & 0.550 \\
\hline Oral contraceptive ${ }^{\#}$ & $8(21)$ & $8(21)$ & 1.000 \\
\hline Pregnancy" & $4(10)$ & $0(0)$ & 0.043 \\
\hline
\end{tabular}

Data are presented as mean \pm SD or $n(\%)$, unless otherwise stated. BMI: body mass index. ${ }^{*}$ : \% of females.

significantly higher in the control compared with the case group, respectively $(44 \%$ versus $22 \%, \mathrm{p}=0.034)$, but not in females $(13 \%$ versus $3 \%, p=0.306)$. 2) All pregnant females were in the case group $(\mathrm{p}=0.043)$ (table 2$)$.

\section{Prevalence of SDB and related symptoms}

The polygraphically assessed RDI was significantly higher in the cases with DVT and/or PE compared with the control group $(p=0.039)$. The mean RDI of 17.8 events $\cdot h^{-1}$ indicates a moderate degree of SDB. The oxygen desaturation index (ODI), mean $\mathrm{Sp}, \mathrm{O}_{2}$ and minimal $\mathrm{Sp}, \mathrm{O}_{2}$ were similar in both groups (table 4). There was a clear predominance of OSA while the occurrence of

\section{TABLE 3 Comorbidities $\#$}

\begin{tabular}{|c|c|c|c|}
\hline & DVT/PE cases & Controls & $\mathrm{p}$-value \\
\hline Arterial hypertension & $31(40)$ & $38(49)$ & 0.257 \\
\hline Coronary artery disease & $10(13)$ & $23(29)$ & 0.011 \\
\hline COPD & $3(4)$ & $8(10)$ & 0.118 \\
\hline Cardiac rhythm & & & 0.306 \\
\hline Sinus rhythm & $71(90)$ & $63(80)$ & \\
\hline Chronic atrial fibrillation & $5(6)$ & $12(15)$ & \\
\hline Intermittent atrial fibrillation & $2(3)$ & $2(3)$ & \\
\hline Paced rhythm & $1(1)$ & $2(3)$ & \\
\hline
\end{tabular}

Data are presented as $\mathrm{n}(\%)$. DVT: deep vein thrombosis; PE: pulmonary embolism; COPD: chronic obstructive pulmonary disease. ${ }^{\#}$ : data sets of 77 case-control pairs.

\begin{tabular}{lccc} 
TABLE 4 & Sleep-disordered breathing and symptoms \\
& DVT/PE cases & Controls & p-value \\
\hline & 82 & 82 & \\
Subjects n & & & \\
Polygraphy & $17.8 \pm 17.9$ & $12.4 \pm 15.2$ & 0.039 \\
$\quad$ RDI events $\cdot h^{-1}$ & $12.0 \pm 17.0$ & $9.6 \pm 15.0$ & 0.349 \\
$\quad$ ODI events $\cdot h^{-1}$ & $93.8 \pm 2.8$ & $94.0 \pm 2.7$ & 0.578 \\
$\quad$ Mean Sp, $\mathrm{O}_{2} \%$ & $78.5 \pm 12.2$ & $81.1 \pm 9.7$ & 0.125 \\
$\quad$ Minimal Sp, $\mathrm{O}_{2} \%$ & & & \\
Symptoms & $50(62)$ & $55(68)$ & 0.411 \\
$\quad$ Snoring n (\%) & & & \\
$\quad$ Epworth Sleepiness Scale score & $4.9 \pm 3.2$ & $6.0 \pm 4.5$ & 0.071 \\
\hline
\end{tabular}

Data are presented as mean $\pm S D$, unless otherwise stated. DVT: deep vein thrombosis; PE: pulmonary embolism; RDI: respiratory disturbance index; ODI: oxygen desaturation index; $\mathrm{Sp}, \mathrm{O}_{2}$ : arterial oxygen saturation. ${ }^{*}$ : data sets of 81 case-control pairs; ": data sets of 77 case-control pairs.

central sleep apnoea with an at least moderate degree (RDI $\geqslant 15$ events $\cdot h^{-1}$ ) was similarly low in cases with DVT and/or PE and controls ( 5 and $6 \%$, respectively; $\mathrm{p}>0.05$ ).

When compared with the controls, the prevalence of an at least mild (RDI $\geqslant 5$ events $\cdot h^{-1}$ ), moderate (RDI $\geqslant 15$ events $\cdot h^{-1}$ ) and severe degree (RDI $\geqslant 30$ events $\cdot h^{-1}$ ) of SDB was increased by $33 \%, 54 \%$ and $83 \%$ in the DVT and/or PE cases $(p=0.013$, $p=0.046$ and $p=0.097$, respectively; fig. 1 ). Sex-specific subanalyses revealed that these differences are based on the significant differences of SDB prevalence between DVT and/or PE cases and controls in females: in females, DVT and/or PE cases had a 51, 208 and $360 \%$ greater prevalence of mild $(p=0.044)$, moderate $(p=0.005)$ and severe $(p=0.023)$ SDB compared with controls, respectively (fig. 2a), while in males, prevalence of at least mild, moderate and severe SDB was similar in all groups $(p=0.111, p=0.823$ and $p=0.786$ respectively; fig. 2b).

The prevalence of witnessed snoring was similar in both groups $(\mathrm{p}=0.411$, table 4$)$. The Epworth Sleepiness Scale score was in the normal range, indicating no excessive daytime sleepiness, and similar in both groups $(p=0.071)$.

\section{Relationship between SDB and thromboembolic events}

To evaluate whether SDB (RDI $\geqslant 15$ events $\cdot h^{-1}$ ) is related to prevalent DVT and/or PE, we performed a multivariate binary regression analysis including established risk factors of thromboembolic events in the model. In the final model, the odds ratio for prevalent DVT and/or PE in individuals with an RDI of $\geqslant 15$ events $\cdot \mathrm{h}^{-1}$ or greater was increased significantly at 2.28 (95\% CI, 1.08-4.85) compared with the reference category (RDI $<15$ events $\cdot h^{-1}$, table 5). Of the known risk factors for thromboembolic events, odds ratios were significantly increased for immobilisation and cancer at 7.67 (95\% CI 2.04-28.91) and 4.25 (95\% CI 1.40-12.91), while there was no statistically significant association of a family history of DVT and/or PE and history of recent surgery or trauma (table 5).

Similar to the comparison of the unadjusted prevalence of SDB $\left(\mathrm{RDI} \geqslant 15\right.$ events $\cdot \mathrm{h}^{-1}$ ) separately in females and males, there is a significant association of SDB (RDI $\geqslant 15$ events $\cdot h^{-1}$ ) and 


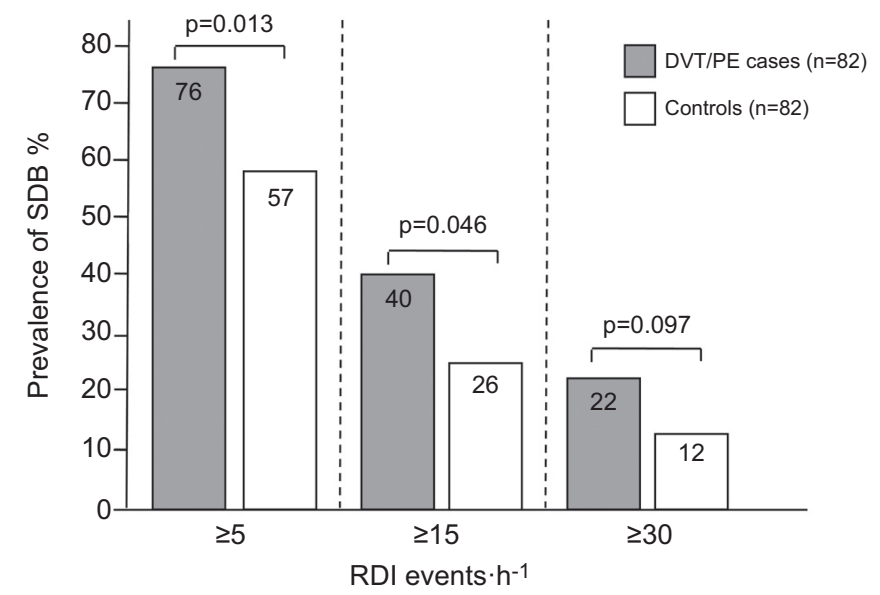

FIGURE 1. Prevalence of sleep-disordered breathing (SDB) in deep vein thrombosis (DVT) and/or pulmonary embolism (PE) cases versus controls. RDI: respiratory disturbance index.

prevalent DVT and/or PE with an odds ratio of 4.14 (95\% CI 1.05-16.36) in females, when accounting for potential confounders. Additional adjustment for pregnancy and coronary artery disease did not significantly change this association (OR $15.2,95 \%$ CI $1.674-138.29 ; p=0.016)$.
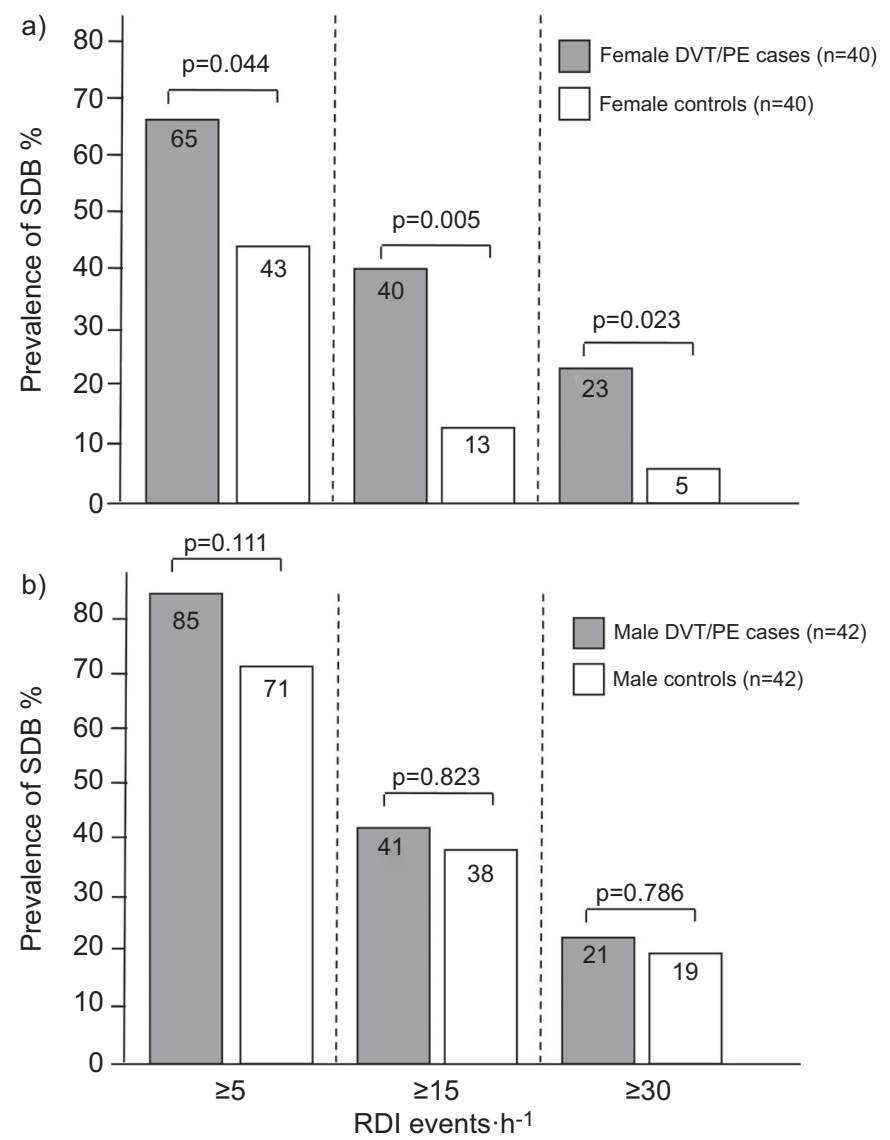

FIGURE 2. Prevalence of sleep-disordered breathing (SDB) in deep vein thrombosis (DVT) and/or pulmonary embolism (PE) cases versus controls. a) Female subjects; b) male subjects. RDI: respiratory disturbance index.

\begin{tabular}{|c|c|c|c|}
\hline $\begin{array}{ll}\text { TABLE } 5 & \text { Multivaria } \\
\text { thromboe }\end{array}$ & inary & $\begin{array}{l}\text { egression analys } \\
\text { ents and potentia }\end{array}$ & $\begin{array}{l}\text { etween } \\
\text { sk factors }\end{array}$ \\
\hline \multirow{2}{*}{ Independent variable } & \multirow[t]{2}{*}{$\mathbf{n}$} & \multicolumn{2}{|c|}{ DVT and/or PE cases } \\
\hline & & OR $(95 \% \mathrm{Cl})$ & $\mathrm{p}$-value \\
\hline Entire sample & 164 & & \\
\hline $\mathrm{SDB}^{\#}$ & & $2.28(1.08-4.85)$ & 0.032 \\
\hline Immobilisation & & $7.67(2.04-28.91)$ & 0.003 \\
\hline Cancer & & $4.25(1.40-12.91)$ & 0.011 \\
\hline Family history of DVT/PE & & $2.63(0.99-7.00)$ & 0.052 \\
\hline Surgery or trauma & & $2.08(0.74-5.87)$ & 0.167 \\
\hline Sub-analysis in females & 80 & & \\
\hline $\mathrm{SDB}^{\#}$ & & $4.14(1.05-16.36)$ & 0.042 \\
\hline Immobilisation & & $11.43(1.09-120.31)$ & 0.042 \\
\hline Cancer & & $2.75(0.58-13.06)$ & 0.205 \\
\hline Family history of DVT/PE & & $2.20(0.52-9.24)$ & 0.282 \\
\hline Surgery or trauma & & $1.61(0.26-9.98)$ & 0.611 \\
\hline Sub-analysis in males & 84 & & \\
\hline $\mathrm{SDB}^{\#}$ & & $1.55(0.57-4.21)$ & 0.391 \\
\hline Immobilisation & & $5.26(0.92-30.15)$ & 0.062 \\
\hline Cancer & & $12.16(1.40-105.35)$ & 0.023 \\
\hline Family history of DVT/PE & & $3.03(0.63-14.57)$ & 0.166 \\
\hline Surgery or trauma & & $2.45(0.63-9.50)$ & 0.194 \\
\hline
\end{tabular}

The multivariate binary regression model included sleep-disordered breathing (SDB), surgery or trauma, immobilisation, cancer, family history of deep vein thrombosis (DVT) and/or pulmonary embolism (PE) as independent variables. \#: respiratory disturbance index $\geqslant 15$ events $\cdot h^{-1}$.

In contrast, in males SDB (RDI $\geqslant 15$ events $\cdot h^{-1}$ ) and prevalent DVT and/or PE are not significantly related (OR 1.55, 95\% CI 0.57-4.21; table 5). After additional adjustment for coronary artery disease, there was a statistically nonsignificant increase of this association (OR 2.78, 95\% CI 0.89-8.72; $\mathrm{p}=0.079$ ).

\section{DISCUSSION}

The present study has given rise to several novel observations. First, in patients with DVT and/or acute PE, the prevalence of an at least moderate degree of SDB is significantly higher compared with age-, BMI- and sex-matched controls. Second, the main type of SDB observed in these patients is OSA. Third, while in females the presence of moderate SDB is significantly associated with prevalent DVT and/or PE independent of established risk factors for thromboembolic events, this association was modest and nonsignificant in males.

We observed that $40 \%$ of cases with DVT and/or PE had an RDI $\geqslant 15$ events $\cdot h^{-1}$. An earlier case series published as a research letter found an even higher prevalence of at least moderate SDB (i.e. 63\%) among 68 patients with DVT and/or PE [2]. However, it is difficult to compare these data with ours as scoring criteria for SDB and patient age, which may both influence the prevalence of SDB, were not given [2]. Another previous report observed a lower prevalence of SDB in patients with venous thromboembolism [3]. In this study, $15.5 \%$ of patients had OSA with an apnoea-hypopnoea index $\geqslant 5$ events $\cdot h^{-1}$. However, this diagnosis was retrospectively ascertained based on file information and thus a considerable number of SDB cases may have 
been missed [3]. In a third study investigating subjects who underwent a CT scan for suspected PE, those with PE had a high rate of a positive Berlin Questionnaire responses, suggesting the presence of SDB (i.e. 65\%) [4].

In the control group without DVT and/or PE, which was closely matched with the index patients for anthropometric variables, the prevalence of SDB was considerably lower (i.e. $26 \%$ ). This is in accordance with the data by EPSTEIN et al. [4] who found that $36 \%$ of their control patients had SDB. The other two preceding studies did not have a control group [2,3]. The prevalence of at least moderate SDB in our hospitalised controls may be considered to be relatively high; however, these figures are usually higher than in a community sample of similar age [7-9]. For instance, BIXLER et al. [8] reported that $12 \%$ of individuals living in the community have an RDI $\geqslant 15$ events $\cdot h^{-1}$.

Previous studies did not report on the type of SDB in DVT and/or PE patients $[2,3,8]$. In our patients with DVT and/or $\mathrm{PE}$, there was a clear predominance of OSA. Only $5 \%$ of them suffered from central sleep apnoea, which is comparable to the proportion of this type of SDB in males of similar age from the community sample [8].

The presence of moderate SDB was significantly associated with prevalent DVT and/or PE independent of established risk factors for thromboembolic events. In line with these findings, EPSTEIN et al. [4] found a significant and independent association between the risk of having SDB and prevalent acute PE (OR 2.78, 95\% CI 1.54-5.03).

In addition, our study provides the first sex-specific subanalyses showing that the association between moderate SDB and prevalent DVT and/or PE is highly significant in females, while this is not the case in males. The reason for this observation is unclear; however, it may be speculated that it is due to a sex difference in endothelial function in patients with SDB. This is supported by data from FAULX et al. [10] who have found that impairment of endothelium-dependent vasodilation is significantly greater in females than in males with SDB. However, it must be admitted that the evidence for this observed sex difference is limited, because many vasoreactivity studies in OSA exclusively enrolled males [11, 12].

Various pathophysiological mechanisms potentially contributing to the emergence of DVT and/or PE in patients with OSA have been described. Platelet activation and aggregation are enhanced in untreated OSA [13]. Probably, such effects are promoted by increased expressions of receptors mediating interactions between platelets themselves and the endothelium $[14,15]$. Furthermore, circulating fibrinogen levels are elevated in ischaemic stroke patients suffering from OSA [16]. In addition, plasminogen activator inhibitor is increased in OSA [17]. Finally, red blood cell counts and erythrocyte aggregability may be increased in OSA in response to nocturnal hypoxia [18, 19]. Taken together, these changes may lead to both enhanced intravascular coagulation and diminished fibrinolysis, and ultimately, to clot formation. Interestingly, pathways associated with a pro-thrombotic potential seem to be up-regulated even at modest levels of SDB [20]. This is also in line with our finding that the odds ratio for DVT and/or PE was already increased in relatively mild cases of SDB (i.e. those with a RDI $>15$ events $\cdot h^{-1}$ ).
It should be acknowledged that the current study has some possible limitations. First, five-channel validated polygraphy [21] was used to detect SDB instead of fully equipped polysomnography. However, the channels used for respiratory analysis were the same as in polysomnography and all sleep studies were visually analysed by an experienced investigator blinded to the clinical status of the patients.

Second, one should be cautious of interpreting our crosssectional data in terms of causality and time course. Since assessment of SDB was performed after the occurrence of PE, we cannot rule out that right heart failure may have triggered SDB. This issue should be addressed by future studies employing for instance randomised interventions with positive airway pressure therapy.

Third, as a consequence of using a "nested" case-control design with a hospitalised control group with a high prevalence of cardiovascular disease, we may have failed to demonstrate a significantly increased prevalence of SDB in male cases with DVT and/or PE compared to controls.

Fourth, the regression models show relatively wide confidence intervals in the sex-specific subanalyses reflecting less study power due to the lower sample size. Therefore, the nonsignificantly increased odds ratio in males with SDB for prevalent DVT and/or PE may reflect a lack of study power rather than a lack of association.

Finally, we did not perform blood analyses to evaluate the patients for the presence of haemostatic changes and/or inherited thrombophilia. However, strengths of our study include the casecontrol design of patients with versus without DVT and/or PE, its prospective nature, and the diagnosis of DVT and/or PE according to established guidelines.

In conclusion, this study could demonstrate that SDB (mainly OSA) occurs more frequently in females with DVT and/or PE than in controls matched for anthropometric variables. Effects were independent of established risk factors for thromboembolic events. To gain further insights into the time course and causality of the relationship between SDB and thromboembolism longitudinal and intervention studies are needed.

\section{SUPPORT STATEMENT}

This study was supported by a research grant from Respironics, Herrsching, Germany.

\section{STATEMENT OF INTEREST}

Statement of interest for M. Arzt and for the study itself can be found at www.erj.ersjournals.com/site/misc/statements.xhtml

\section{REFERENCES}

1 von Kanel R, Dimsdale JE. Hemostatic alterations in patients with obstructive sleep apnea and the implications for cardiovascular disease. Chest 2003; 124: 1956-1967.

2 Arnulf I, Merino-Andreu M, Perrier A, et al. Obstructive sleep apnea and venous thromboembolism. JAMA 2002; 287: 2655-2656.

3 Bosanquet JP, Bade BC, Zia MF, et al. Patients with venous thromboembolism appear to have higher prevalence of obstructive sleep apnea than the general population. Clin Appl Thromb Hemost 2011; 17: E119-E124. 
4 Epstein MD, Segal LN, Ibrahim SM, et al. Snoring and the risk of obstructive sleep apnea in patients with pulmonary embolism. Sleep 2010; 33: 1069-1074.

5 Hach-Wunderle V. Interdisziplinäre S2-Leitlinie: Diagnostik und Therapie der Bein- und Beckenvenenthrombose und der Lungenembolie. [Interdisciplinary S2 guidelines. Diagnosis and therapy in bone and deep venous thrombosis and pulmonary embolism]. Hamostaseologie 2005; 25: 237-238.

6 Iber C, American Academy of Sleep Medicine. The AASM Manual for the Scoring of Sleep and Associated Events: Rules, Terminology and Technical Specifications. Westchester, American Academy of Sleep Medicine, 2007.

7 Bixler EO, Vgontzas AN, Lin HM, et al. Prevalence of sleepdisordered breathing in women: effects of gender. Am J Respir Crit Care Med 2001; 163: 608-613.

8 Bixler EO, Vgontzas AN, Ten Have T, et al. Effects of age on sleep apnea in men: I. Prevalence and severity. Am J Respir Crit Care Med 1998; 157: 144-148.

9 Young T, Palta M, Dempsey J, et al. The occurrence of sleepdisordered breathing among middle-aged adults. $N$ Engl J Med 1993; 328: 1230-1235.

10 Faulx MD, Larkin EK, Hoit BD, et al. Sex influences endothelial function in sleep-disordered breathing. Sleep 2004; 27: 1113-1120.

11 Ip MS, Tse HF, Lam B, et al. Endothelial function in obstructive sleep apnea and response to treatment. Am J Respir Crit Care Med 2004; 169: 348-353.

12 Kraiczi H, Caidahl K, Samuelsson A, et al. Impairment of vascular endothelial function and left ventricular filling: association with the severity of apnea-induced hypoxemia during sleep. Chest 2001; 119: 1085-1091.

13 Bokinsky G, Miller M, Ault K, et al. Spontaneous platelet activation and aggregation during obstructive sleep apnea and its response to therapy with nasal continuous positive airway pressure. A preliminary investigation. Chest 1995; 108: 625-630.

14 El Solh AA, Akinnusi ME, Berim IG, et al. Hemostatic implications of endothelial cell apoptosis in obstructive sleep apnea. Sleep Breath 2008; 12: 331-337.

15 Kobayashi K, Nishimura $Y$, Shimada T, et al. Effect of continuous positive airway pressure on soluble CD40 ligand in patients with obstructive sleep apnea syndrome. Chest 2006; 129: 632-637.

16 Wessendorf TE, Thilmann AF, Wang YM, et al. Fibrinogen levels and obstructive sleep apnea in ischemic stroke. Am J Respir Crit Care Med 2000; 162: 2039-2042.

17 Rangemark C, Hedner JA, Carlson JT, et al. Platelet function and fibrinolytic activity in hypertensive and normotensive sleep apnea patients. Sleep 1995; 18: 188-194.

18 Hoffstein V, Herridge M, Mateika S, et al. Hematocrit levels in sleep apnea. Chest 1994; 106: 787-791.

19 Peled N, Kassirer M, Kramer MR, et al. Increased erythrocyte adhesiveness and aggregation in obstructive sleep apnea syndrome. Thromb Res 2008; 121: 631-636.

20 Mehra R, Xu F, Babineau DC, et al. Sleep-disordered breathing and prothrombotic biomarkers: cross-sectional results of the Cleveland Family Study. Am J Respir Crit Care Med 2010; 182: 826-833.

21 DGSM. S3-Leitlinie unerholsamer Schlaf/Schlafstörungen [S3 guideline for unrestorative sleep/sleep disturbances]. Somnologie 2009; 13: Suppl. 2, 4-160. 